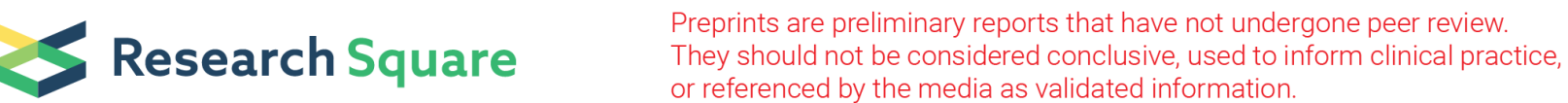

\section{Role of Liver Biopsy in Management of Liver Diseases Following End of the Interferon Era: Experience of A Tertiary Referral Centre}

Nermine A Ehsan

National Liver Institute: Menoufia University National Liver Institute

Maha M Elsabaawy

National Liver Institute: Menoufia University National Liver Institute

Dina M Sweed

National Liver Institute: Menoufia University National Liver Institute

Esraa A Karman

National Liver Institute: Menoufia University National Liver Institute

Eman Abdelsameea ( $\nabla$ eabdelsameea@liver-eg.org )

Menoufia University National Liver Institute https://orcid.org/0000-0002-3225-7164

Anwar A Mohamed

National Liver Institute: Menoufia University National Liver Institute

\section{Research Article}

Keywords: Liver biopsy, liver diseases, etiological diagnosis, histopathological evaluation.

Posted Date: October 27th, 2021

DOI: https://doi.org/10.21203/rs.3.rs-935002/v1

License: (1) This work is licensed under a Creative Commons Attribution 4.0 International License.

Read Full License 


\section{Abstract}

Background: Liver biopsy (LB) is the cornerstone in the management of patients with liver diseases. However, a lot of queries had emerged about its role following the end of the interferon era. The aim of this study was to re-evaluate the current role of LB in the diagnosis of liver diseases.

Methods: All patients who had underwent LB at the department of Hepatology, National Liver Institute, from January 2015 through December 2018 were recruited. Indications for LB, pathology reports, and medical records of all cases were retrieved, reviewed and statistically analyzed.

Results: A total of 275 liver biopsies were collected, 191 males and 84 females with mean age $41.22 \pm 13.36$ years. Etiological diagnosis made by histopathological evaluation was 48 drug induced liver injury (DILI), 42 Non-alcoholic fatty liver disease (NAFLD), 34 chronic hepatitis B, or C with cholestasis, 29 autoimmune hepatitis, 34 primary sclerosing cholangitis, 13 primary biliary cholangitis, 7 autoimmune overlap syndrome, 13 active bilharziasis, 10 Wilson's disease. Minor number of cases were diagnosed by different other etiologies. Initial diagnosis was made by liver biopsy and confirmed by clinical response and laboratory findings.

Conclusion: Liver biopsy is still considered as the gold standard diagnostic measure of different liver diseases representing an integral component of management decisions in hepatology.

\section{Introduction}

There is no doubt that histopathological examination of liver biopsy (LB) is valuable in providing informative data about liver tissue with proper evaluation of the degree of hepatic injury and fibrosis (1). The integration of clinical and laboratory data in conjunction with liver histological examination aid the clinicians towards better evaluation of liver diseases (2). Despite the invasiveness, cost, reported complications, along with the inter-, and intra-observer misconceptions, LB had kept its role as a sole final key for most unresolved liver disorders (3). In the interferon era, LB was mandatory for hepatitis C virus (HCV) treatment decisions (4). Nowadays, with the emerging generations of direct acting antiviral drugs (DAAs) against HCV, LB is claimed to be limitedly used (5). Additionally, in advanced diseases, development of liver cirrhosis might veil the original causative pathology sufficing with the clinical, laboratory, and radiological data (6). Moreover, the ongoing impressive development of noninvasive radiological measures estimating hepatic fibrosis had weakened the need of LB (7). All these novelties together might pose an owed question about the rationale of performing LB. Accordingly, reevaluation of the role LB as an undying effectual diagnostic approach in the era of DAAs was the impulse of designing this study.

\section{Patients And Methods}

Patients' Selection: This observational longitudinal study was conducted on patients who had undergone LB at the National liver Institute (NLI), Menoufia University, Egypt, from January 2015 through December 
2018. Unexplained elevations of liver transaminases and or hepatic cholestasis, along with unexplained liver related conditions were mostly the indications of LB. All patients were subjected to thorough history taking and detailed clinical examination. Complete liver panel tests including transaminases, bilirubin, albumin, alkaline phosphatase, gamma glutamyl transferase, international normalized ratio (INR), Complete blood picture, serum creatinine, blood urea and Alpha-fetoprotein. Viral markers as HCV Ab, HBsAg, HBc IgM, HBclgG were done to all cases. While HIV Ab, EBV IgM, HSVIgM, HDV IgM were done only if needed.

Other laboratory investigations aiming at helping diagnosis were done only whn indicated like serum total IgG level, ANA, ASMA, and LKM-1, AMA Anti-neutrophil cytoplasmic (ANCA) and anti-double strand DNA, angiotensin converting enzyme, copper and ceruloplasmin, and 24hour urinary copper. HCV-RNA was performed to all HCV infected patients using real time PCR technique using Roche COBAS AmpliPrep/COBAS TaqMan HCV assay (Roche Diagnostics, Germany). HBV-DNA levels were obtained for all HBV infected patients using real time PCR technique using Roche COBAS AmpliPrep/COBAS TaqMan HBV Test (Roche Diagnostics, Germany). The protocol was approved by the Institutional Review Board of the National Liver Institute, Menoufia University. This study conforms to the ethical guidelines of the 1975 Declaration of Helsinki.

Radiologically: Abdominal ultrasound and Doppler on portal and hepatic veins with special emphasis on determining liver echogenicity, portal vein patency, spleen size and fluid collection and renal echogenicity were performed. If other radiological investigations data were done if needed like triphasic spiral CT or Magnetic resonance imaging (MRI) on the abdomen, Magnetic resonance pancreatography (MRCP), chest X-ray.

Liver biopsy (LB): Percutaneous ultrasound or CT guided LB was taken (core not less than $1 \mathrm{~cm}$, taken intercostally from right lobe of the liver) under both local (xylocaine), and light sedation (Midazolam 2-3 mg intravenously). A written informed consent was taken from all cases or their relatives. Patients on antiplatelet drugs and anticoagulants stopped taking these medications one week before LB. The patient sited in the supine position with the right hand placed under the head, venous access was established in the left arm. The routine use of ultrasound was helpful in establishing the best location for biopsy, visualizing the liver during inspiration and expiration and identifying neighboring organs (i.e., lung, kidney, gut) that may be at risk of inadvertent puncture. The location for biopsy was marked clearly and then the area was prepped and draped in a sterile fashion (8). One percent lidocaine (Xylocaine) was injected over the upper border of a rib avoiding the intercostal nerve and vessels that run along the lower border. $\mathrm{A}$ small incision was made, and the patient held his or her breath cutting needle was applied to obtain a biopsy either the manual Tru-Cut needle or the spring-loaded automatic needles. LB was fixed in formalin and paraffin embedded. The patient was kept under observation for at 6hours, then discharged if no complications reported.

Histopathological examination: LB was subjected to routine processing for histopathological examination. All tissue sections were stained with hematoxylin \& eosin, masson trichrome, orcein and 
Perls' stain. Liver tissues that revealed biliary injury, bile ducts and ductules were visualized by CK7 immunostaining. Two experienced liver histopathologists evaluated the histological changes in liver sections unaware to the clinical diagnosis.

The exclusion criteria in these series were patients with extrahepatic biliary obstruction, hydatid cyst, ascites and those with severe coagulopathy.

Statistical analysis: Data were collected, tabulated, statistically analyzed using an IBM personal computer with Statistical Package of Social Science (SPSS) version 20 where the following statistics were applied. Descriptive statistics, in which quantitative data was presented in the form of mean ( ), standard deviation (SD), range, and qualitative data had been presented in the form of numbers and percentages.

\section{Results}

Patients' characteristics: A total of 275 liver biopsies were collected, 191 males and 84 females with mean age $41.22 \pm 13.36$.

Different etiological diagnosis were obtained from histopathological examination and were classified as follows: DILI comprising 48/275(17.45\%) cases followed by biliary cholangitis 47 (17.09\%) (Primary sclerosing cholangitis, primary biliary cholangitis), 42 (15.27\%) non-alcoholic fatty liver disease (NAFLD), 36 (13.09\%) autoimmune hepatic liver disease (Autoimmune hepatitis (AlH), autoimmune overlap), 34 (12.36\%) chronic viral hepatitis infection (HCV \& HBV), 11 (4\%) sarcoidosis. Thirteen cases were active bilharziasis were responsible for $4.72 \%$ of all indications for LB.

In the current study, the major indications for LB were DILI and was responsible for $17.45 \%$ of all indications of LB in our series. Despite LB was not indicated in DILI cases proved clinically, the main indication of LB was in atypical cases or cases denied drug history. LB had established the diagnosis of DILI in patients taken a common prescribed drug, herbals, or pesticides exposure. The reported pathological features were either combined hepatitis and cholestasis $21.5 \%$, chronic hepatitis in $21.5 \%$, intrahepatic cholestasis $21.5 \%$, combined cholestasis and steatosis in $10.6 \%$, acute hepatitis $5.3 \%$, syncytial hepatitis $5.3 \%$, or steatohepatitis in $5.3 \%$. Eosinophils rich infiltrate was a nearly constant finding in most of cases.

LB played an initial diagnostic role in five cases of early-stage biliary disease ( $10.6 \%$ of biliary cholangitis cases) on which the patient presented by elevated liver enzymes with normal or mild elevated bilirubin level. In addition, in AlH cases, LB had a key role in establishing the diagnosis in three cases $(8.3 \%$ of AlH cases) presenting with negative autoantibodies and in raising the possibility of associated biliary overlap disease in six cases ( $16.7 \%$ of AlH cases) apart from defining the grade and stage of disease.

In NAFLD cases, LB confirmed the clinical diagnosis and differentiated NAFLD from NASH in 35 cases (83.3\% of NAFLD cases). However, the remaining 7 cases (16.7\% of NAFLD cases), the patients were slim, 
had no diabetes or insulin resistance and normal abdominal sonography. The liver pathology showed a mild steatosis $(20 \%)$, ballooning or lobular inflammation which raised the diagnosis of lean NAFLD.

LB remained the gold standard in diagnosis of granulomatous liver disease and defining the causative agents (bilharzial ova in 13 cases and numerable unpaired granulomas suspicious for sarcoidosis in 11 cases).

Table 2 showed most common drugs encountered in DILI. It was evident in our study group that nonsteroidal anti-inflammatory drugs diclofenac was the most common cause of DILI followed by herbal medicine, Amoxicillin-clavulanic acid, halothane anesthetic agent, amiodarone, pesticides, and disinfectants. Rare cases of DILI necessitating LB were isoniazid, atorvastatin, estrogen and carbimazole.

The reported complications after LB in the current study were illustrated in table 3. Pain was the most evident minor complications and was present in $93.09 \%$ patients. Analgesic agent was required in $71 / 275(25.18 \%)$ of cases to control pain. Major complications were rare and subsequently seen in 5 $(1.8 \%)$ cases with no death. Bleeding occurred in 2 cases with one case was severe required blood transfusion and conservative treatment was enough. The other complications were one case $(0.36 \%)$ for either biliary leak, pneumothorax or sepsis.

\section{Discussion}

Since the advent of DAAs, the number of daily done liver biopsies (LB) had been considerably lessened (9). Additionally, the suggested high accuracy of the non-invasive measures had imposed a big query on the current significance of LB in liver disease diagnosis. Confidently, LB is never to be outdated, and there will be always a substantial role for histopathological examination in solving diagnostic dilemmas (8). In this unique study we had investigated the current diagnostic needs of LB after quitting interferon therapy which was mainly dependent on LB finding adoption of the new DAAs.

Drug induced liver injury (DILI) is one of the most common indications of LB as its diagnosis is mainly based on exclusion of all other etiologies of liver diseases which is practically impossible without the print of LB (10).

Accordingly, DILI had attained the highest number of cases that had performed LB and included in the current study (17.45\%). The presence of combined hepatitis and cholestasis, eosinophilia, along with centrilobular necrosis are the most prominent features of DILI. However, a wide range of histopathological patterns had been identified in DILI cases according to the offending drug. Picture of acute hepatitis, acute liver failure, steatohepatitis, granulomatous hepatitis and eventually some cases develop chronic hepatitis leading to liver cirrhosis (11). Once cirrhosis had been developed the recognition of the primary pathology is significantly concealed. In a recent Egyptian study, non-steroidal antiinflammatory (NSAID) drugs was reported as the first offending drug in DILI occurrence (10). Similarly, 
were the results of the current study, where NSAID represented about $20 \%$ of cases, with $8 \%$ for herbal preparations, and $5 \%$ each of amoxycillin clavulanic acid and halothane.

Unrecognized drugs also represent $5 \%$ of cases which is a threatening alarm for more social orientation of drug hazards along with more governmental censorship for limitation of over the counter drugs.

NAFLD is considered the evolving most common cause of liver diseases nowadays. In the current study NAFLD was the second prevalent indication of LB 42(12.75\%). The global prevalence of NAFLD in the Middle East is high and affects about $31.8 \%$ of adult population (12). Based on the strong association between NAFLD and the metabolic syndrome a new nomination had been emerged to be metabolic associated fatty liver disease (MAFLD) (13). Hepatic steatosis more than $5 \%$ along with ballooning, lobular inflammation and even fibrosis are the main characteristics of NASH (14). Despite the availability of many non-invasive measures for evaluation of steatosis and specifically fibrosis, LB is still the most accurate distinguishing maneuver of NASH from NAFLD (15). In MAFLD, the linkage between liver fibrosis and cardiovascular risks is well identified denoting higher rates of morbidity and mortality (13). Accordingly, LB is still mandated in this area either as a diagnostic or a prognostic measure. In spite of the necessity of LB in NAFLD diagnosis in all international guidelines, the only obstacle prohibiting generalization of this concept is the patient's refusal. More efforts should be wielded to delineate and eliminate all patients' fairs and illusions about LB.

In the current study, chronic viral hepatitis infections were the fourth cause of performing LB $(12.36 \%)$. Five cases with hepatitis C, 25 cases with hepatitis B virus infection and 4 cases with dual hepatitis B and C infection.

The unprecedented Egyptian campaign for treating HCV, was greatly appreciated all over the world. Since the emergence of direct acting antiviral drugs (DAAs) against HCV, the number of performed LBs had remarkably lessened. Unlike interferon therapy of HCV, DAAs had limited the reliance on LB as a prerequisite of treatment decisions (7). However, LB is still indicated in chronic HCV cases that showed poor sustained viral response in order to define a concomitant disease either iron overload, steatosis or granuloma that could resist the therapy.

In chronic active hepatitis B virus (HBV) infection, antiviral therapy can often be initiated without the need of LB. However, the difficulty in determining disease activity and fibrosis by serologic and biochemical data in chronic HBV makes LB an important tool in the management of a subset of patients (7). Demonstration of the degree of fibrosis and/or inflammation or associated concomitant disease in LB is a decisive factor for both treatment indications (16).

In patients who have HBV DNA 2,000 IU/ml and at least moderate fibrosis, treatment may be initiated even if ALT levels are normal (17). Moreover, the debatable queries about either to treat or not those with HBV chronic active infections could be partially resolved relying on histopathological examination of LB (18). 
The treatment of dual HCV and HBV infections are challenging, as treating HCV with DAAs had been associated with increase of HBV-DNA levels in patients with positive HBsAg. In some cases, elevated ALT and even liver failure may develop in these conditions (19-20). Accordingly, LB might be of significance in determining the most prevailing infection and determining treatment priorities.

In our series $\mathrm{AlH}$ patients are representing about $13 \% \%$ of the whole cohort. From our data, LB is the most trustworthy diagnostic measure of $\mathrm{AlH}$ included in all old and recent scoring systems of $\mathrm{AlH}$ recommended by international guidelines (21). Remission of AlH is considered in those who develop normalization of both serum transaminases and IgG (22). However, normalization of either of them mandates reliance on histopathological examination of LB to assure remission (21). LB should be performed also for immunosuppression resistant cases for the possibility of overlap syndrome (which was detected in $2.45 \%$ of cases in the current study). Recognition of the prevailing pathology in overlap syndrome could not be assured without LB. Nevertheless, progression to liver cirrhosis in those patients remains the obstacle masquerading the prime pathology (21).

In the current series biliary tract diseases especially biliary cholangitis (PSC, PBC) are considered important causes of liver disease. They represent $17.9 \%$ of liver biopsies in the National liver disease. Biliary diseases should be taken in consideration as an important cause of liver disease as prolonged biliary inflammation leads to many serious complications like cholangitis, biliary abscesses and liver cirrhosis which is a fast devastating incident in this context (23). Biliary cirrhosis is distinguished to have a hastier course rather than other liver pathologies, a fact mandating early diagnosis and rapid management (24). Unlike PBC, LB is not mandated for most of PSC cases as magnetic resonance cholangiography (MRCP) and or endoscopic retrograde cholangiography (ERCP) remains the most reliable diagnostic measure (25). Cases with small duct PSC or overlap syndromes with PSC were the only cases relying on LB results (26). While destruction of small bile ducts is the most pathognomonic histopathological feature of PBC, the presence of onion skin fibrosis is the devastating sign of PSC (27).

Granulomatous hepatitis is the most diagnostic feature of sarcoidosis representing $4 \%$ of the current study cases. Diagnosis of cases with hepatic sarcoidosis might be challenging for the non-specific presentations. LB might be the last resolution for this dilemma. Non caseating numerable unpaired granuloma and intrahepatic cholestasis were found to be the prevailing histological features of hepatic sarcoidosis (28). However, sometimes the distinction from early non-caseating TB granuloma is frustrating necessitating resorting to more distinctive special stains (28).

The need of LB for recognition of hepatic Schistosomiasis was reported in $4.72 \%$ of current study cases. Schistosomal non-cirrhotic portal hypertension was the most prevalent diagnostic need for LB with the characteristic hepatic granuloma (29). Thanks to the governmental efforts, the prevalence of hepatic schistosomiasis had been dramatically ameliorated in Egypt (30).

Long ago, LB is only needed for exclusion of other causes of liver disease rather than diagnosis of Wilson disease (WD). The required dried weight liver tissue for proper diagnosis of WD is a cumbersome against LB. the wide range of histopathological presentations might also be another con (31). In accord, LB was 
needed in only in 10 cases (3.63\%) in this research that were with clinical and laboratory inconclusive data.

Despite rarity, liver might be the seat of non-hepatotropic viral infections. Sometimes LB might be the leading suggestive measure of non-hepatotropic viral infections while searching of cause of the liver injury in a puzzling case (32). Epstein-Barr Virus (EBV) had affected 6 cases while cytomegalovirus (CMV) had affected 4 cases of patients included in the current study. The presence of lobular disarray, nuclear inclusion bodies, micro-abscesses, and lobular lymphocytic infiltration are the main histopathological features suggestive of CMV in LB (32).

In EBV, portal tracts are massively intruded by atypical lymphocytes and plasma cells. Intra-sinusoidal lymphocytosis often has a characteristic "Indian-file" appearance (32).

Seven cases of Dubin-Johnson syndrome were reported in the current study. Surprisingly these cases might present up to fifties with unexplained conjugated hyperbilirubinemia and normal transaminases. Grossly the liver appeared black with the absence of anti-MRP2 on immunohistochemical staining of liver tissue (33).

Liver abscess is a rare indications for liver biopsy in our study and represents less than $2 \%$. In Cryptogenic pyogenic liver abscess is present in $60 \%$ of cases whenever there is no obvious identifiable risk factor (34). Most pyogenic liver abscesses are located in the right lobe of the liver because of portal vein anatomy, more hepatic mass and dense bile canaliculi in this lobe (35). This is also prominent in our series as 5 of 6 liver abscesses were found to be located in the right lobe of the liver. The imaging of the liver plays a corner stone in helping detection of liver abscess. Abdominal ultrasound and CT scan can help in diagnosis of $50 \%$ of cases (36). Nevertheless, LB is requested only in cases of early hepatic masses before evident liquefaction along with atypical imaging criteria.

Solitary cases of latent congenital hepatic fibrosis, amyloidosis, hemochromatosis, hepatic amoebiasis, malaria, polyarteritis nodosa, myeloproliferative disease was also reported in the current series.

The imaging guided LB had limited the associated risks of this interventional theoretically invasive maneuver. Regarding LB complications in the current study, pain requiring analgesia was the main complain (25.18\%). Major complications represented only less than $1.18 \%$ with no reported mortalities. Bleeding $(0.7 \%)$, biliary leak $(03 \%)$, sepsis $(0.03 \%)$ and pneumothorax $(0.3 \%)$ were reported without either surgical interventions or unfavorable outcomes. Similarly, Elsenberg and his colleagues, had denoted the occurrence of pain even mild discomfort following LB in 84\% (37). Also, Kose et al had reported that post-LB pain requiring analgesia was present in $19.8 \%$ and major complications in $1.15 \%$. Major complications in their study included pneumothorax in $0.17 \%$, haemobilia in $0.08 \%$ and hematoma in $0.9 \%$ ) and absence of other complications like abscess, bacteremia, organ perforation and death (38). Also, Govender et al reported serious complications in a rate of $1.7 \%$ in their series in the form of pneumothorax, symptomatic hematoma and pseudoaneurysm and no deaths (39). The mortality rate attributed to the procedure is estimated in one per 10 thousand biopsies and is usually secondary to 
bleeding; this is even lower when the biopsy is not performed for evaluation of liver tumors (40). The reported higher rates of safety had denied any claims of high-risk probabilities.

In the current study, the complete absence of what was known as intra-observer variations denoting the highly professional skillful pathology team, adding more reliability to the maneuver.

\section{Conclusion}

Conclusively the tapered decline in LB requests upon the advent of DAAs treating HCV infections along with the higher rates of accuracy of laboratory and imaging diagnostic measures had been questioned lately. However, LB will remain the golden key of lots of hepatic diagnostic dilemmas. Additionally, the higher reported safety rates might help in gaining more confidence among refusal parties. Currently, the mapping of liver diseases prevalence had been significantly changed. While MAFLD, DILI, and AlH are the main indications of LB nowadays, the need of LB in viral infections had become substantially limited.

Figure 1 demonstrated the most frequent etiological diagnosis made by LB histopathological evaluation.

Liver tissue examination from cases of DILI exhibited microvesicular steatosis, frequent apoptotic bodies, cholestatic rosettes and inflammatory infiltrate rich in eosinophils in portal tracts (Fig.1a). Primary sclerosing cholangitis and primary biliary cholangitis were clearly visualized by masson trichrome stain and CK7 immunostaining of destructive bile ducts and ductules and showed bile ductular proliferation and bilirubin stasis (Fig. 1b,1c). Liver sections exhibited prominent plasmalymphocytic infiltrate together with plasma cell clusters were consistent with autoimmune hepatitis (Fig. 1d). Autoimmune overlap syndrome displayed combined hepatitis and biliary injury as seen in Fig. 1e. HCV-Patients who did not receive DAAs and had developed elevated liver enzymes, their LB revealed features of chronic liver disease with variable stage of fibrosis (Fig.1f).

Figure 2 showed less common diagnosis made by LB examination. Non-hepatotropic viruses (EpsteinBarr or cytomegaloviruses) exhibited sinusoidal beaded lymphocytes, viral inclusions and microabcesses (Fig. 2a). Wilson's disease was identified histologically by orcein stain positive copper associated protein deposits (Fig. 2b). Indications for LB in these cases were 10 (3.63\%) cases for each. Seven cases were diagnosed as Dubbin-Johnson syndrome as the LB examination revealed preserved architecture and normal looking hepatocytes except for perivenular zone exhibited bilirubin stasis in the cytoplasm of hepatocytes (Fig. 2C). Six cases were diagnosed as liver abscess. Four cases were diagnosed as resolving acute hepatitis, that were identified by the hypertrophied Kupffer cells, frequent apoptotic bodies and resetting of hepatocytes (Fig. 2d). Very rare cases presented as a solitary case each like latent congenital hepatic fibrosis (Fig 2e), amyloidosis, hemochromatosis (Fig 2f), hepatic amoebiasis, malaria, polyarteritis nodosa, myeloproliferative disease.

\section{Declarations}

\section{Funding:}


(in case of funding): The study did not receive any funding.

\section{Conflict of Interest:}

no conflict of interest

\section{Ethical approval:}

The study was performed in accordance with the moral framework of the 1975 Declaration of Helsinki, and it was revised and agreed upon by the Research Ethics Committee of the National Liver Institute, Menoufia University.

* Informed consent. Informed written consent was obtained from all participants

\section{References}

1. Tapper EB, Lok AS. Use of Liver Imaging and Biopsy in Clinical Practice. N Engl J Med 2017; 377:756.

2. Ovchinsky N, Moreira RK, Lefkowitch JH, Lavine JE. Liver biopsy in modern clinical practice: a pediatric point-of-view. Adv Anat Pathol. 2012;19(4):250-262.

3. Pandey N, Hoilat GJ, John S. Liver Biopsy. [Updated 2020 Dec 9]. In: StatPearls [Internet]. Treasure Island (FL): StatPearls Publishing; 2020 Jan-. Available from: https://www.ncbi.nlm.nih.gov/books/NBK470567/.

4. Sherman M, Shafran S, Burak K, Doucette K, Wong W, Girgrah N, Yoshida E, Renner E, Wong P, Deschênes M. Management of chronic hepatitis C: consensus guidelines. Can J Gastroenterol. 2007 Jun;21 Suppl C (Suppl C):25C-34C.

5. Gonzalez HC, Jafri SM, Gordon SC. Role of liver biopsy in the era of direct-acting antivirals. Curr Gastroenterol Rep. 2013 Feb;15(2):307.

6. Bosman FT, Carneiro F, Hruban RH, Theise ND (2010) Hepatocellular carcinoma. WHO Classification of tumours of the Digestive System, 4th Edition, International Agency for Research on Cancer. Lyon. 2010: 348-402.

7. Misdraji Changing indications for liver biopsy: viral hepatitis. Diagnostic Histopathology 2014 ;20:119-124.

8. Boyd A, Cain O,Chauhan A, Jame G, Webb GJ. Medical liver biopsy: background, indications, procedure and histopathology Frontline Gastroenterol . 2020;11(1):40-47.

9. Mansour H, Zaky S, El Kassas M, Mamdouh H, Esmat G. Evaluating the effect of direct-acting agents on liver fibrosis, by real-time elastography, Fibroscan and FIB4 score in chronic HCV patients 20193 (1): 237-245.

10. Alhaddad O, Elsabaawy M, Abdelsameea E, et al. Presentations, Causes and Outcomes of DrugInduced Liver Injury in Egypt. Sci Rep. 2020 Mar 20;10(1):5124. 
11. Björnsson ES, Bergmann OM, Björnsson HK, Kvaran RB, Sigurdur Olafssolncidence, presentation, and outcomes in patients with drug-induced liver injury in the general population of Iceland. Gastroenterology. 2013;144(7):1419-25.

12. Younossi ZM, Marchesini G, Pinto-Cortez H, Petta S. Epidemiology of Nonalcoholic Fatty Liver Disease and Nonalcoholic Steatohepatitis: Implications for Liver Transplantation. Transplantation 2019;103(1):22-27.

13. Fouad, Y, Waked, I, Bollipo, S, Gomaa, A, Ajlouni, Y, Attia, D. What's in a name? Renaming 'NAFLD' to 'MAFLD'. Liver Int. 2020; 40: 1254- 1261.

14. Chalasani N, Younossi Z, Lavine JE,et al. The diagnosis and management of nonalcoholic fatty liver disease: Practice guidance from the American Association for the Study of Liver Diseases. 2018 Jan;67(1):328-357.

15. Yeh MM, Brunt EM. Pathological features of fatty liver disease. Gastroenterology. 2014; 147:754764.

16. Gameel KH, M. Elsabaawy M, Naguib M, et al. Role of Liver Biopsy in HBV Infected Egyptian Patients: A New Insight. British Journal of Medicine and Medical Research, ISSN: 2231-0614: 19, 2.

17. EASL 2017 Clinical Practice Guidelines on the management of hepatitis B virus infection. J Hepatol. 2017;67(2):370-398.

18. Metwally, K., Elsabaawy, M., Abdel-Samiee, M., Morad, W., Ehsan, N., Abdelsameea, E. (2020). FIB-5 versus FIB-4 index for assessment of hepatic fibrosis in chronic hepatitis $B$ affected patients. Clinical and experimental hepatology, 6(4), 335-338.

19. Bersoff-Matcha SJ, Cao K, Jason M, Ajao A, Jones SC, MeyerT, Brinker A. Hepatitis B Virus Reactivation Associated With Direct-Acting Antiviral Therapy for Chronic Hepatitis C Virus: A Review of Cases Reported to the U.S. Food and Drug Administration Adverse Event Reporting System. Ann Intern Med 2017; 166: 792-798.

20. Belperio PS, Shahoumian TA, Mole LA, Backus LI. Evaluation of hepatitis B reactivation among 62,920 veterans treated with oral hepatitis $C$ antivirals. Hepatology 2017; 66: 27-36

21. Mack, C.L., Adams, D., Assis, D.N., et al. Diagnosis and Management of Autoimmune Hepatitis in Adults and Children: 2019 Practice Guidance and Guidelines From the American Association for the Study of Liver Diseases. Hepatology 2020, 72: 671-722.

22. Tiniakos DG, Brain JG, Bury YA Tiniakos. Role of Histopathology in Autoimmune Hepatitis. Dig Dis. 2015;33 Suppl 2:53-64.

23. Lindor, K.D., Bowlus, C.L., Boyer, J., Levy, C., Mayo, M. Primary Biliary Cholangitis: 2018 Practice Guidance from the American Association for the Study of Liver Diseases. Hepatology 2019, 69: 394419.

24. Purohit T, Cappell MS. Primary biliary cirrhosis: Pathophysiology, clinical presentation and therapy. World J Hepatol. 2015;7(7):926-941.

25. Sirpal S, Chandok N. Primary sclerosing cholangitis: diagnostic and management challenges. Clin Exp Gastroenterol. 2017; 10:265-273. 
26. Igarashi G, Endo T, Mikami K, et al. Two Cases of Primary Sclerosing Cholangitis Overlapping with Autoimmune Hepatitis in Adults. Intern Med. 2017;56(5):509-515. doi:10.2169/internalmedicine.56.7633.

27. Tabibian JH, Bowlus CL. Primary sclerosing cholangitis: A review and update. Liver Res. 2017;1(4):221-230. doi:10.1016/ j. livres.2017.12.002.

28. Doppalapudi H, Markus JT, Parekh U. Granulomatous Hepatitis. [Updated 2020 Nov 3]. In: StatPearls [Internet]. Treasure Island (FL): StatPearls Publishing; 2020 Jan-. Available from: https://www.ncbi.nlm.nih.gov/books/NBK564315/

29. Parris V, Michie $K$, Andrews T, et al. Schistosomiasis japonicum diagnosed on liver biopsy in a patient with hepatitis B co-infection: a case report. J Med Case Rep. 2014; 8:45. Published 2014 Feb 12. doi:10.1186/1752-1947-8-45.

30. Rollinson D, Knopp S, Levitz S, Stothard JR, Tchuem Tchuenté LA. Time to set the agenda for schistosomiasis elimination. Acta Trop 2013; 128: 423-440.

31. Oe S, Honma Y, Yabuki K, et al. Importance of a Liver Biopsy in the Management of Wilson Disease. Intern Med. 2020;59(1):77-81.

32. Dancygier H. Viral Infections by Nonhepatotropic Viruses. Clinical Hepatology. 2010;823-830.

33. Devgun MS, El-Nujumi AM, O'Dowd GJ, Barbu V, Poupon R. Noval mutations in the Dubin-Johnson syndrome gene ABCC2/MRP2 and associated biochemical changes. Ann Clin Biochem. 2012; 49:609-12.

34. Malik AA, Bari SU, Rouf KA, et al. Pyogenic liver abscess: changing patterns in approach. World J Gastrointest Surg 2010; 2:395-401.

35. Serraino C, Elia C, Bracco C, et al. Characteristics and management of pyogenic liver abscess: A European experience. Medicine (Baltimore). 2018 May;97(19):1-6

36. Lo JZ, Leow JJ, Ng PL, et al. Predictors of therapy failure in a series of 741 adult pyogenic liver abscesses. J Hepatobiliary Pancreat Sci. 2015; 22:156-165.

37. Eisenberg E, Konopniki M, Veitsman E, Kramskay R, Gaitini D, BaruchY. Prevalence and characteristics of pain induced by percutaneous liver biopsy. Anesth Analg 2003; 96:1392-1396, table of content

38. Kose S, Ersan G, Tatar B, Adar P, Sengel BE. Evaluation of Percutaneous Liver Biopsy Complications in Patients with Chronic Viral Hepatitis. Eurasian J Med. 2015 Oct; 47(3):161-4.

39. Govender P, Jonas MM, Alomari Al, et al. Sonography-guided percutaneous liver biopsies in children. AJR J Roentgenol 2013; 201: 645-50.

40. West J, Card TR. Reduced mortality rates following elective percutaneous liver biopsies. Gastroenterology. 2010; 139:1230-7.

\section{Tables}

Table 1: Indications of liver biopsy at the National liver institute, Egypt 


\begin{tabular}{|c|c|}
\hline Indications for LB & $\mathbf{N}(\%)$ \\
\hline DILI & $48(17.45)$ \\
\hline Biliary diseases & $\underline{47}(\underline{17.09})$ \\
\hline PSC & $34(12.36)$ \\
\hline PBC & $13(4.72)$ \\
\hline NAFLD & $42(15.27)$ \\
\hline Autoimmune liver disease & $36(13.09)$ \\
\hline AlH & $29(10.54)$ \\
\hline Autoimmune overlap syndrome & $7(2.54)$ \\
\hline Chronic viral hepatitis & $\underline{34}(\underline{12.36})$ \\
\hline Chronic HBV & $25(9.09)$ \\
\hline Chronic HCV & $5(1.81)$ \\
\hline Dual HCV and HBV infection & $4(1.45)$ \\
\hline Sarcoidosis & $11(4)$ \\
\hline Active bilharziasis & $13(4.72)$ \\
\hline Wilson's disease & $10(3.63)$ \\
\hline Non hepatotropic viruses & 10 (3.63). \\
\hline CMV & $6(2.18)$ \\
\hline EBV & $4(1.45)$ \\
\hline Dubbin Johnson syndrome & $7(2.54)$ \\
\hline Liver abscess & $6(2.18)$ \\
\hline Resolving acute hepatitis & $4(1.45)$ \\
\hline Rare cases & $7(2.54)$ \\
\hline Latent congenital hepatic fibrosis & $1(0.36)$ \\
\hline \multicolumn{2}{|l|}{ Amyloidosis } \\
\hline Hemochromatosis & $1(0.36)$ \\
\hline Hepatic amoebiasis & $1(0.36)$ \\
\hline Malaria & $1(0.36)$ \\
\hline Polyarteritis Nodosa & $1(0.36)$ \\
\hline Myeloproliferative disease & $1(0.36)$ \\
\hline
\end{tabular}


Abbreviations; DILI drug induced liver injury, NAFLD nonalcoholic fatty liver disease, HCV; hepatitis C virus, HBV; hepatitis B virus ,AlH; autoimmune hepatitis, PSC ;primary sclerosing cholangitis, PBC; primary biliary cholangitis, CMV; cytomegalovirus, EBV; Epstein Barr virus.

Table 2: Most implicated drugs in drug induced liver injury in the present study

\begin{tabular}{|ll|}
\hline Drugs (LB in only 48 cases) & $\mathbf{N}(\%)$ \\
\hline Non-steroidal anti-inflammatory drug & $10(20.3)$ \\
\hline Herbal remedies & $8(16.6)$ \\
\hline Amoxicillin clavulanic acid & $5(10.4)$ \\
\hline Halothane anesthesia & $5(10.4)$ \\
\hline Unrecognized type of drug intake & $5(10.4)$ \\
\hline Amiodarone & $4(8.3)$ \\
\hline Pesticides \&disinfectants & $4(8.3)$ \\
\hline Oral contraceptives & $2(4.1)$ \\
\hline Isoniazid & $2(4.1)$ \\
\hline Lipid lowering agent atorvastatin & $2(4.1)$ \\
\hline Carbimazole & $1(2.08)$ \\
\hline
\end{tabular}

Table 3: Complications of LB. 


\begin{tabular}{|ll|}
\hline Complications of LB & $\mathrm{N}(\%)$ \\
\hline Minor complications & \\
Pain requiring analgesia & $71(25.18)$ \\
\hline Major complications & $5(1.8)$ \\
\hline Bleeding & $2(0.72)$ \\
\hline Massive requiring blood transfusions & $1(0.36)$ \\
\hline Minimal (conservative treatment) & $1(0.36)$ \\
\hline Biliary leak & $1(0.36)$ \\
\hline Sepsis & $1(0.36)$ \\
\hline Pneumothorax & $1(0.36)$ \\
\hline Death & 0 \\
\hline
\end{tabular}

Figures 

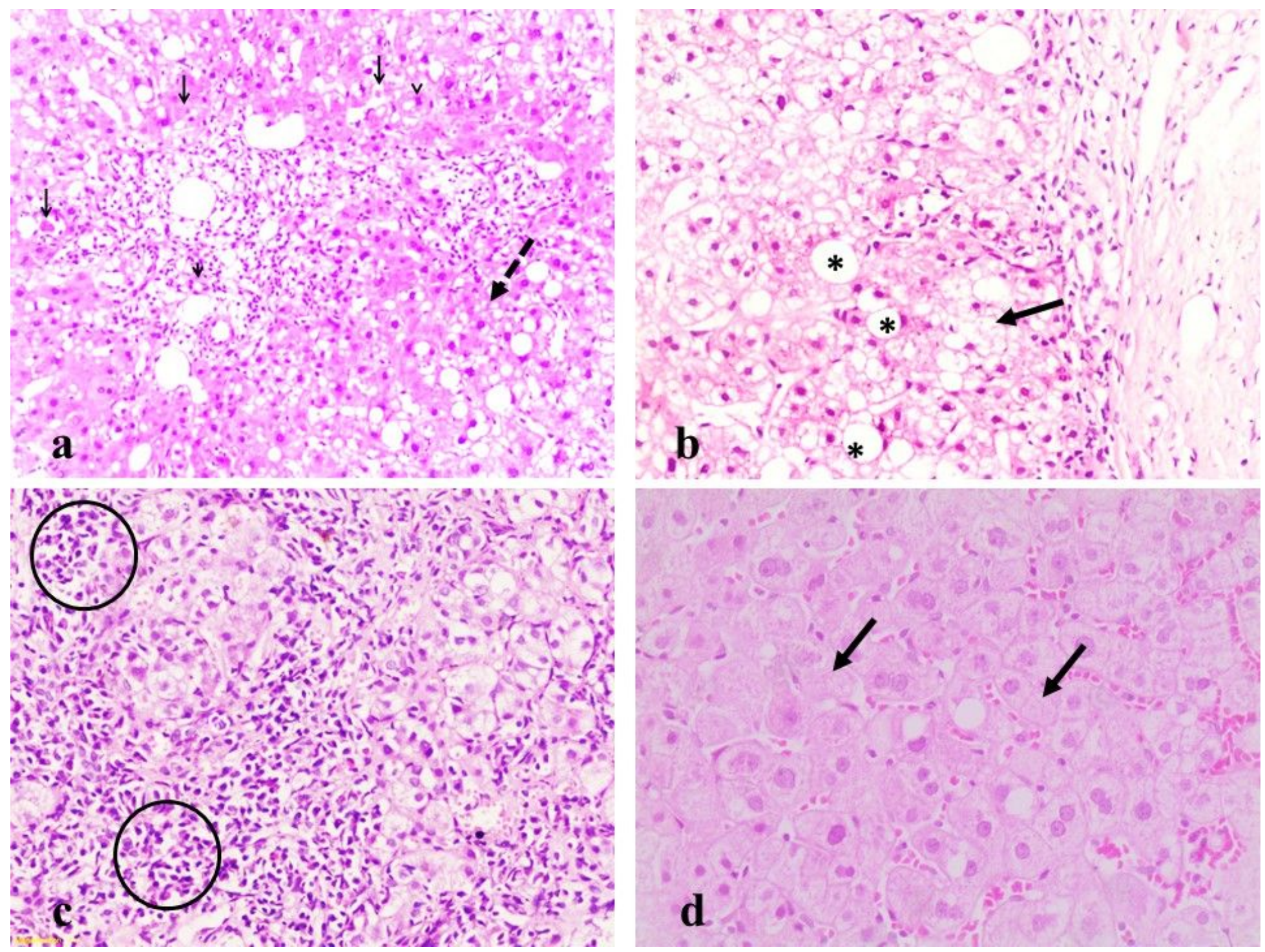

\section{Figure 1}

Figure 1 demonstrated the most frequent etiological diagnosis made by LB histopathological evaluation. Liver tissue examination from cases of DILI exhibited microvesicular steatosis, frequent apoptotic bodies, cholestatic rosettes and inflammatory infiltrate rich in eosinophils in portal tracts (Fig.1a). Primary sclerosing cholangitis and primary biliary cholangitis were clearly visualized by masson trichrome stain and CK7 immunostaining of destructive bile ducts and ductules and showed bile ductular proliferation and bilirubin stasis (Fig. 1b,1 C). Liver sections exhibited prominent plasmalymphocytic infiltrate together with plasma cell clusters were consistent with autoimmune hepatitis (Fig. 1d). Autoimmune overlap syndrome displayed combined hepatitis and biliary injury as seen in Fig. 1e. HCV-Patients who did not receive DAAs and had developed elevated liver enzymes, their LB revealed features of chronic liver disease with variable stage of fibrosis (Fig.1f). 

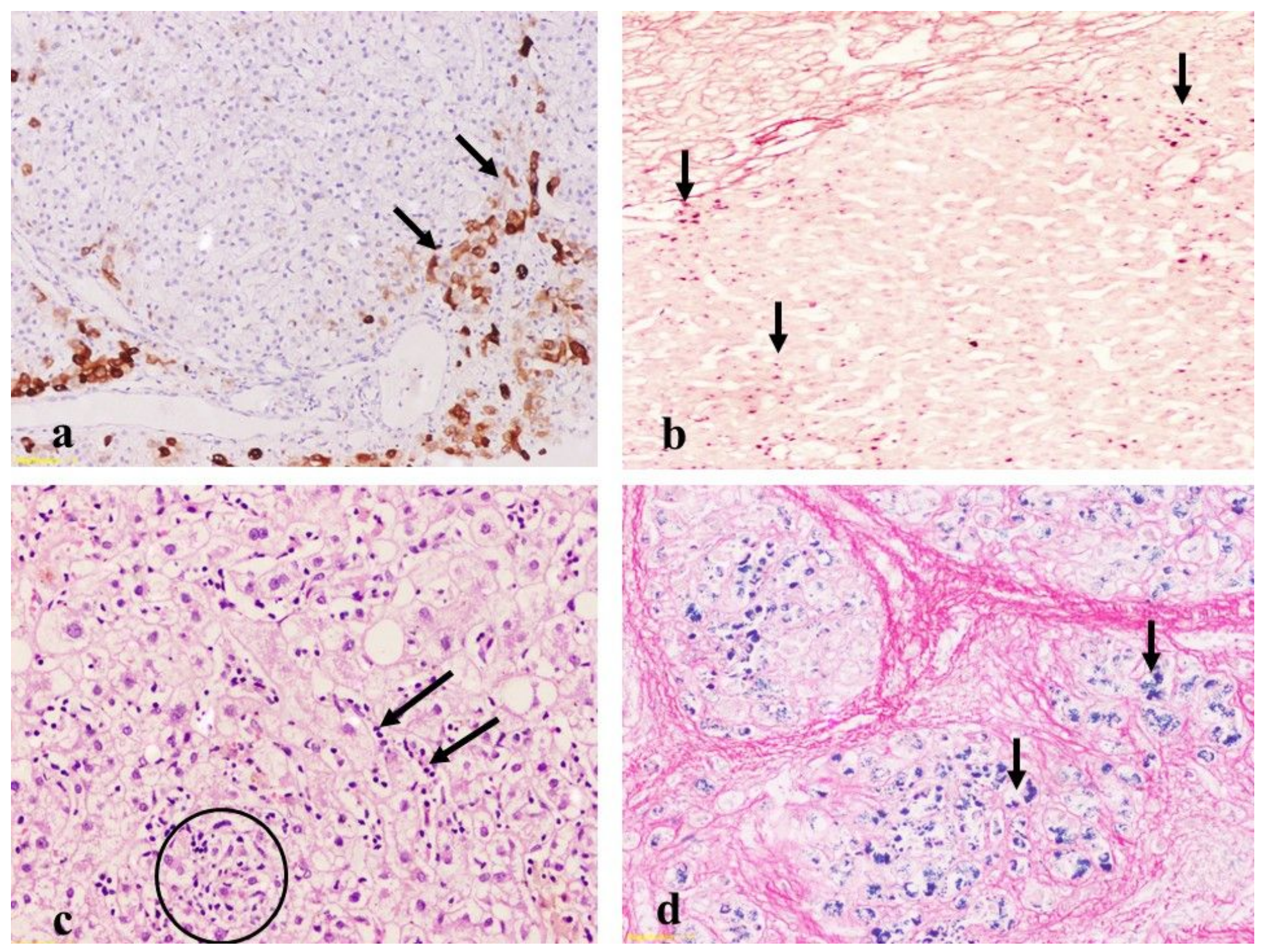

Figure 2

Figure 2 showed less common diagnosis made by LB examination. Non-hepatotropic viruses (EpsteinBarr or cytomegaloviruses) exhibited sinusoidal beaded lymphocytes, viral inclusions and microabcesses (Fig. 2a). Wilson's disease was identified histologically by orcein stain positive copper associated protein deposits (Fig. 2b). Indications for LB in these cases were 10 (3.63\%) cases for each. Seven cases were diagnosed as Dubbin-Johnson syndrome as the LB examination revealed preserved architecture and normal looking hepatocytes except for perivenular zone exhibited bilirubin stasis in the cytoplasm of hepatocytes (Fig. 2c). Six cases were diagnosed as liver abscess. Four cases were diagnosed as resolving acute hepatitis, that were identified by the hypertrophied Kupffer cells, frequent apoptotic bodies and resetting of hepatocytes (Fig. 2d). Very rare cases presented as a solitary case each like latent congenital hepatic fibrosis (Fig 2e), amyloidosis, hemochromatosis (Fig 2f), hepatic amoebiasis, malaria, polyarteritis nodosa, myeloproliferative disease. 